\title{
Emergency Department Airway Management for Status Asthmaticus With Respiratory Failure
}

\author{
Haley T Godwin, Megan L Fix, Olesya Baker, Troy Madsen, Ron M Walls, and \\ Calvin A Brown III
}

\begin{abstract}
BACKGROUND: Data are limited regarding current practice and outcomes for emergency department airway management in status asthmaticus. This paper describes the foremost methods and outcomes of airway management in patients in the emergency department who required intubation for status asthmaticus. METHODS: We analyzed all intubations with a primary indication of asthma over a 3-y period (January 1, 2016 to December 31, 2018) using the National Emergency Airway Registry (NEAR), a 25-center, prospective, observational registry of emergency department intubations. We report the incidence of intubations for asthma, methods and medications used, devices used, peri-intubation adverse events, and intubation success and failures using univariate descriptive statistics and cluster-adjusted incidence with 95\% CI. RESULTS: A total of 19,071 encounters were recorded during the study period, with 14,517 patients intubated for medical indications. Of those, $173(1.2 \%, 95 \%$ CI $0.9-1.6)$ were intubated for asthma. The firstattempt success rate was $90.8 \%$ (95\% CI 81.9-95.5), and overall intubation success was $100 \%$. Compared to the medical registry as a whole, patients with asthma were more likely to undergo rapid-sequence intubation (96.5\% [95\% CI 92.9-98.3] vs 80.8\% [95\% CI 75.1-82.5]), preoxygenation with bi-level positive airway pressure (BPAP) $(62.9 \%$ [95\% CI 49.6-74.6] vs $13.5 \%$ (95\% CI 10.4-16.9]), and induction with ketamine (51.8\% [95\% CI 30.6-71.4] vs $11.6 \%$ [95\% CI 7.616.8]). The adverse event rate in the patients with asthma was $12.14 \%$ (95\% CI 8.1-17.9) compared to $11.93 \%(95 \%$ CI 9.79-14.12) in the medical registry. CONCLUSIONS: Status asthmaticus accounted for about $1 \%$ of emergent medical intubations. The majority of patients were intubated using rapid-sequence intubation after preoxygenation with BPAP and induction with ketamine, with the latter 2 practices being much more common for emergent intubations for status asthmaticus than for other medical indications. Key words: asthma; emergency department; airway. [Respir Care 2020;65(12):1904-1907. (C) 2020 Daedalus Enterprises]
\end{abstract}

\section{Introduction}

There are $>25$ million Americans living with asthma, and $>45 \%$ of individuals with asthma experience at least one asthma attack in a 12-month period. ${ }^{1-2}$ Asthma accounts

\footnotetext{
Ms Godwin, Dr Fix, and Dr Madsen are affiliated with the School of Medicine, University of Utah, Salt Lake City, Utah. Drs Baker, Walls, and Brown are affiliated with Brigham and Women's Hospital, Harvard Medical School, Boston, Massachusetts.

The authors have disclosed no conflicts of interest.

Correspondence: Haley T Godwin, 30 N 1900 E, Salt Lake City, UT 84132. E-mail: Haley.godwin@hsc.utah.edu.
}

DOI: $10.4187 /$ respcare. 07723 for nearly two million visits to the emergency department each year. ${ }^{3-4}$ Worldwide, it is estimated that approximately 250,000 premature deaths can be attributed to asthma, with 3,500 of those occurring in the United States. ${ }^{3,5}$ Up to $20 \%$ of patients presenting to the emergency department with asthma require admission to the ICU, and approximately one third of these patients require intubation. ${ }^{6}$

There are no national guidelines for airway management in status asthmaticus. Data on emergent emergency department intubations for patients with asthma are sparse and focus largely on mortality rates and not airway management techniques or practices. Complication rates have been reported at up to $45 \%$ of emergent asthma intubations, with mortality rates generally low but ranging up to $20 \%$. $^{4}$ The last multicenter surveillance of emergency department intubations reported that nearly $3 \%$ of all intubations were 


\section{Emergency Airway Management for Status Asthmaticus With Respiratory Failure}

performed for severe asthma, but techniques and outcomes in these patients were not reported. ${ }^{7}$ Ketamine has been reported to have bronchodilatory properties and has been advocated for emergency intubation for status asthmaticus. ${ }^{8}$

The National Emergency Airway Registry (NEAR) is a prospective, multi-center, observational, intubation registry coordinated through the Department of Emergency Medicine at Brigham and Women's Hospital in Boston, Massachusetts (http://www.nearstudy.net, Accessed April $21,2020)$. The purpose of our investigation is to describe current airway management techniques, devices, and outcomes for patients undergoing intubation for severe asthma using multicenter surveillance from the NEAR project.

\section{Methods}

We analyzed all intubations with a primary indication of asthma over a 3-y period (January 1, 2016 to December 31, 2018) using the NEAR database. Institutional review board approval was obtained at each participating site. Registrywide reporting compliance was $>90 \%$. We report the incidence of intubations for asthma, methods and medications used, device(s) used, success rates, and peri-intubation adverse events, using univariate descriptive statistics and cluster-adjusted incidence rates with $95 \%$ CI.

\section{Results}

A total of 19,071 encounters were recorded during the study period, with 14,517 patients intubated for medical indications. Of those, 173 (1.2\% [95\% CI 0.9-1.6]) had asthma as the primary indication for intubation. Table 1 provides a comparison of demographics of asthma

Table 1. Characteristics for Patient Populations With and Without Asthma

\begin{tabular}{lcc}
\hline \hline & With Asthma & Without Asthma \\
\hline Patient characteristics & & \\
Age, y & $43.3(39.9-46.7)$ & $55.3(51.5-59.2)$ \\
Gender (female), \% & $54.3(43.1-65.1)$ & $37.2(34.8-39.6)$ \\
Any difficult airway & $43.4(35.2-51.9)$ & $54.2(46.3-60.6)$ \\
$\quad$ characteristics, \% & & \\
Level of intubator experience & & \\
Emergency medicine PGY1 & $10.7(4.9-21.6)$ & $12.7(7.5-20.4)$ \\
Emergency medicine PGY2 & $31.4(19.6-46.2)$ & $32.9(23.1-43.7)$ \\
Emergency medicine PGY3 & $40.2(23.3-59.9)$ & $38.8(24.5-54.43)$ \\
Emergency medicine PGY4 & $6.5(2.2-17.7)$ & $8.3(5.0-13.2)$ \\
Emergency medicine PGY $\geq 5$ & $5.2(2.1-15.8)$ & $3.4(1.4-8.1)$ \\
$\quad$ or fellow & & \\
Emergency medicine attending & $5.3(2.1-13.0)$ & $3.8(2.5-5.8)$ \\
$\quad$ physician & & \\
\hline Data are presented as means (95\% CI). & & \\
PGY = postgraduate year & & \\
\hline
\end{tabular}

\section{QUICK LOOK}

\section{Current knowledge}

Current knowledge on the foremost methods and outcomes of intubation for emergent asthma is extremely limited. No descriptive study has ever analyzed a registry to detail methods and outcomes, despite rates of asthma rising worldwide.

\section{What this paper contributes to our knowledge}

Intubation for asthma was uncommon within our registry. When required, the majority of patients were intubated using rapid-sequence intubation after preoxygenation with bi-level positive airway pressure and induction with ketamine, all practices that are unique to asthma. The overall success rate in this population was $100 \%$, indicating that the above method appears to be an effective approach to intubation for emergent cases of asthma.

patients in the medically indicated intubation registry. Approximately $63 \%$ of patients (56 of 89 ) with a reported method of preoxygenation were preoxygenated with BPAP. Most patients were intubated using video laryngoscopy $(n=113,66.9 \%)$. Rapid-sequence intubation was used in $96.5 \%$ of cases. For these, $50.9 \%(n=85,95 \%$ CI 30.1-71.4) were induced with ketamine, followed by etomidate at $48.5 \%(n=81,95 \%$ CI 27.8-69.7). By comparison, use of ketamine as the induction agent for rapidsequence intubation for patients intubated for a medical indication other than asthma was $10.59 \%(n=1,214,95 \%$ CI 6.87-15.99). For neuromuscular blockade, providers used succinylcholine for $44.9 \%$ of patients and rocuronium for $55.1 \%$, similar to the rest of the registry for all medically indicated intubations. There was an overall adverse event rate of $12.1 \%$ in the population with asthma. The most common adverse events reported were hypoxia $(n=14,8.1 \%)$ and hypotension $(n=5,2.9 \%)$. There were no barotrauma adverse events recorded. A total of 157 patients were intubated on the first attempt, 12 on the second attempt, 3 on the third attempt, 1 on the fourth attempt, and 1 on the fifth attempt. First-attempt intubation success was $90.8 \%$, and ultimate success was $100 \%$. The first-attempt success rate did not differ significantly between devices, with $92.0 \%$ for video laryngoscopy and $87.5 \%$ for direct laryngoscopy $(P=.43)$. There was no difference in first-attempt success stratified by induction agent. One successful rescue cricothyrotomy was performed. Compared to the registry as a whole, patients with asthma were more likely to undergo rapid-sequence intubation (96.5\% [95\% CI 92.9-98.3] vs 80.8\% [95\% CI 75.1-82.5]) (Fig. 1), preoxygenation with BPAP (62.9\% 


\section{Emergency Airway Management for Status Asthmaticus With Respiratory Failure}

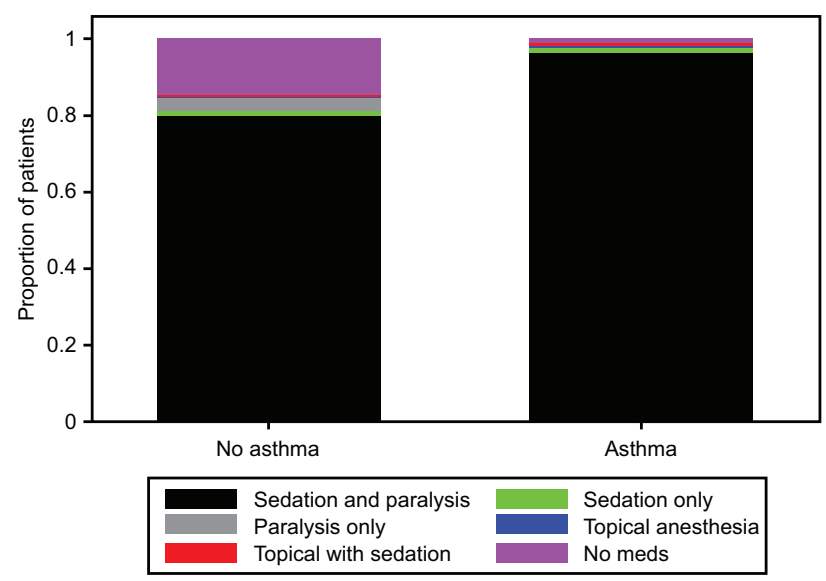

Fig. 1. Method of intubation for patient populations with and without asthma.

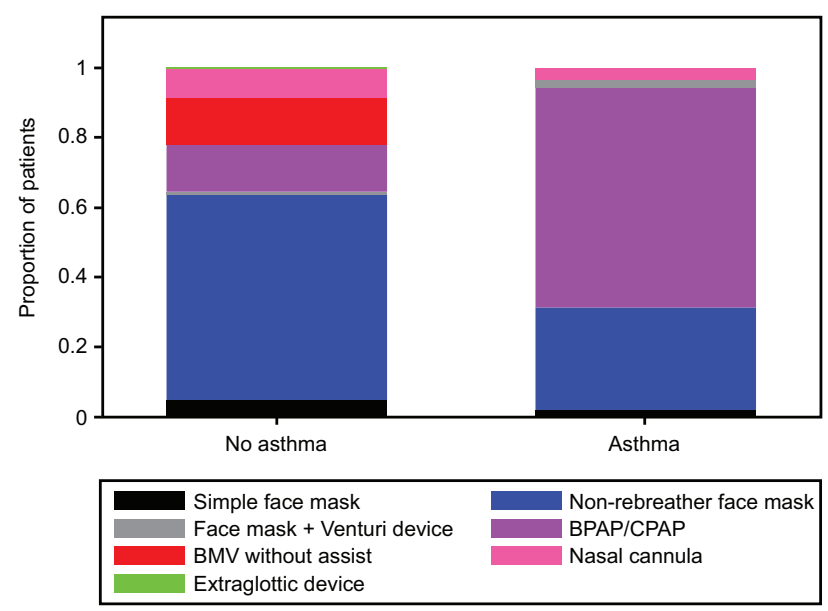

Fig. 2. Pre-oxygenation method in subjects with and without asthma. $\mathrm{BVM}=$ bag-valve-mask, BPAP = bilevel positive airway pressure.

[95\% CI 49.6-74.6] vs $13.5 \%$ [95\% CI 10.4-16.9]) (Fig. $2)$, and induction with ketamine $(51.8 \%$ [95\% CI 30.671.4] vs $11.6 \%$ [95\% CI 7.6-16.8]) (Fig. 3).

\section{Discussion}

Status asthmaticus is a relatively uncommon indication for emergency intubation in our cohort of nearly 20,000 intubations in the emergency department. Intubations for patients with asthma shared many characteristics with the larger database, including video laryngoscope as the primary device, use of rocuronium versus succinylcholine as a paralytic agent (Fig. 4), adverse event incidence, firstattempt success rate, and overall success rate. Notably, intubations for patients with asthma differed from the general population in the use of rapid-sequence intubation, BPAP for preoxygenation, and ketamine for induction.

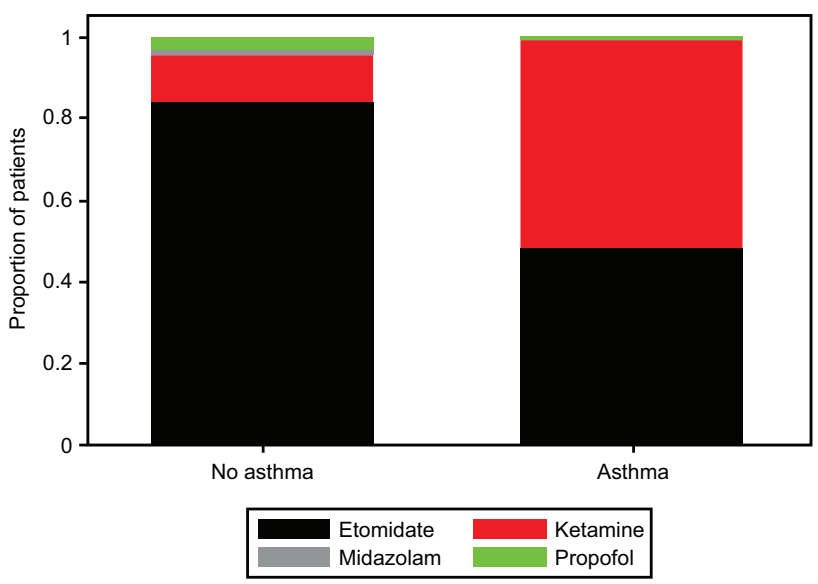

Fig. 3. Induction agent in patient populations with and without asthma.

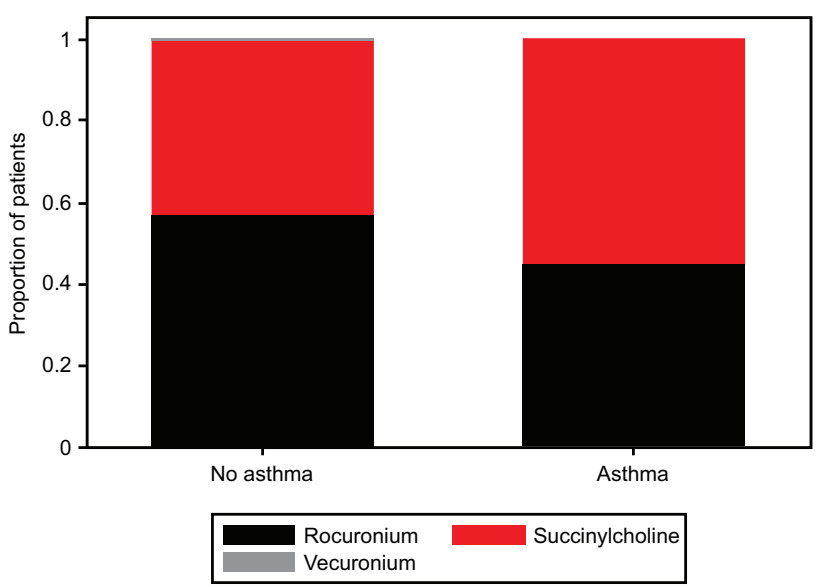

Fig. 4. Paralytic agent in patient populations with and without asthma.

The use of rapid-sequence intubation in patients with asthma was significantly higher than the use of rapidsequence intubation in the general population. The percentage of patients with any difficulty airway characteristic was higher in the general population compared to those with asthma. This lower rate of anatomic difficulty, younger average age, and the need to achieve successful intubation promptly in the context of rapid oxyhemoglobin desaturation likely explains the higher rate of rapid-sequence intubation use in this population (Fig. 1).

Preoxygenation with BPAP was used in nearly two thirds of intubations for patients with asthma. This is significantly higher than the use of BPAP for preoxygenation in patients intubated for medical indications other than asthma. BPAP is commonly used as part of first-line treatment in the emergency department for severe asthma as a method to increase ventilation and reduce work of 


\section{Emergency Airway Management for Status Asthmaticus With Respiratory Failure}

breathing. It is unclear if the use of preoxygenation with BPAP for intubation of patients with asthma is indicative of routine use as a first-line treatment, or if it was a deliberate decision by providers to achieve preoxygenation in preparation for intubation.

Finally, the use of ketamine as an induction agent in the population of patients with asthma who required intubation was significantly higher than the general population. The increased use of ketamine in this population is not surprising considering the purported bronchodilatory effects of ketamine. ${ }^{8}$ Although previously reported to be used in nearly $90 \%$ of rapid-sequence intubations overall, etomidate was used in only half of intubations for patients with asthma, a consequence of the increased use of ketamine in this population. ${ }^{9}$ After multivariable regression controlling for variables that impact first-attempt success in patients in the emergency department, we found no difference in firstattempt success between etomidate and ketamine in patients with asthma, suggesting that either drug is appropriate in this patient population.

Compared to the rest of the registry, first-attempt success and ultimate intubation success were similar. The absence of any adverse events related to barotrauma is reassuring, but this may be a result of small overall numbers, underreporting, or clinically occult barotrauma that may have been diagnosed later in the patient's course of positivepressure ventilation.

These descriptive data show that the approach to emergency airway management for patients with asthma differs from that of the general population in the emergency department. Our results suggest that rapid onset of apnea and robust preoxygenation, more often using positive pressure, are management principles unique to this population and could be the basis for asthma-specific intubation guidelines that could be validated with a clinical trial. Although our observational findings show clinical equipoise between etomidate and ketamine, dosing was not controlled. Additionally, a standard approach that involves preoxygenation with BPAP may be preferred to treat asthma because a patient's increased work of breathing can make preoxygenation with ambient pressure challenging. However, optimal BPAP settings and duration would need to be controlled to understand the best approach. These data lay an important early foundation, and refining a protocolized approach to treat severe asthma requires more multicenter data or a randomized trial.

\section{Conclusions}

Status asthmaticus accounts for a small minority of intubations performed for medical indications. Most patients requiring intubation for asthma are intubated using rapidsequence intubation after preoxygenation with BPAP and induction with ketamine. The frequent use of BPAP for preoxygenation and ketamine induction differentiate intubation for patients with asthma from intubation for other medical indications. Overall intubation success rate in this population was $100 \%$, indicating that the above method is an effective approach to intubate in cases of emergent asthma.

\section{REFERENCES}

1. Akinbami LJ, Moorman JE, Liu X. Asthma prevalence health care use and mortality: United States 2005-2009. Natl Health Stat Report 2011; 12(32):1-14.

2. To T, Stanojevic S, Moores G, Gershon AS, Bateman ED, Cruz AA, Boulet LP. Global asthma prevalence in adults: findings from the crosssectional world health survey. BMC Pub Health 2012;12:204.

3. Ram S, Zhang W, Williams M, Pengetnze Y. Predicting asthma-related emergency department visits using big data. IEEE J Biomed Health Inform 2015;19(4):1216-1223.

4. Brenner B, Corbridge T, Kazzi A. Intubation and mechanical ventilation of the asthmatic patient in respiratory failure. Proc Am Thorac Soc 2009;6(4):371-379.

5. D’Amato G, Vitale C, Molino A, Stanziola A, Sanduzzi A, Vatrella A, et al. Asthma-related deaths. Multidiscip Resp Med 2016;11:37.

6. Zimmerman JL, Dellinger RP, Shah AN, Taylor RW. Endotracheal intubation and mechanical ventilation in severe asthma. Crit Care Med 1993;21(11):1727-1730.

7. Walls RM, Brown CA, Bair AE, Pallin DJ. Emergency airway management: a multi-center report of 8937 emergency department intubations. J Emerg Med 2011;41(4):347-354.

8. L'Hommedieu CS, Arens JJ. The use of ketamine for the emergency intubation of patients with status asthmaticus. Ann Emerg Med 1987;16 (5):568-571.

9. Brown CA, Bair AE, Pallin DJ, Walls RM, NEAR III Investigators. Techniques, success, and adverse events of emergency department adult intubations. Ann Emerg Med 2015;65(4):363-370. 